\title{
Excitation of Neutron Star Oscillations by an Orbiting Particle
}

\author{
Johannes Ruoff( ${ }^{(1,2)}$, Pablo Laguna ${ }^{(2)}$ and Jorge Pullin ${ }^{(2)}$ \\ (1) Institut für Astronomie und Astrophysik \\ Universität Tübingen, D-72076 Tübingen, Germany \\ (2) Center for Gravitational Physics \& Geometry \\ Penn State University, University Park, PA 16802, USA
}

\begin{abstract}
The excitation of neutron stars is expected to be an important source of gravitational radiation. Of fundamental importance is then to investigate mechanisms that trigger oscillations in neutron stars in order to characterize the emitted radiation. We present results from a numerical study of the even-parity gravitational radiation generated from a particle orbiting a neutron star. We focus our investigation on those conditions on the orbital parameters that favor the excitation of $w$-modes. We find that, for astrophysically realistic conditions, there is practically no $w$-mode contribution to the emitted radiation. Only for particles with ultra-relativistic orbital speeds $\geq 0.9 c$, the $w$-mode does significantly contribute to the total emitted gravitational energy. We also stress the importance of setting consistent initial configurations.
\end{abstract}

\section{INTRODUCTION}

Neutron stars as a source of gravitational radiation have been under investigation for over 30 years, with the foundation developed by the work of Thorne and Campolattaro [1]. The radiation emitted by an excited non-rotating neutron star basically consists of a superposition of the characteristic oscillatory modes of the star 2 - 4 . Those modes fall into two categories. One class are spacetime modes, such as the $w$-modes and the trapped modes for very compact star. The other class consist of fluid modes, such as the $f$-mode and the $p$-modes which also exist in Newtonian stars. Due to the emission of gravitational waves, both types of modes are damped, with the spacetime modes much stronger than the fluid modes.

Recent studies of the time evolution of these oscillatory modes [5, 6 have shown that generic, but somewhat unphysical, initial data can excite the first $w$-mode, the $f$-mode, and a few $p$-modes. However, the strength of these modes depends crucially on the particular choice of initial data. Conformally flat initial data, for instance, has the effect of almost completely suppressing the excitation of $w$-modes [7], whereas the $f$-mode is generally present. Work by Andersson and Kokkotas [8] shows that, by extracting the frequencies and damping times of the first $w$-mode and the $f$-mode, one could in principle determine physical parameters of the neutron star such as mass, radius and even the equation of state. Of course, the success in the determination of such physical parameters will depend on the particular modes present in the signal and the noise level.

The issue of interest for the work in this paper is whether astrophysically motivated initial neutron star perturbations are able to excite the $w$-modes, so that accurate determination of the mode parameters could be possible. One of the most likely events that may excite strong oscillations is the birth of the neutron star. However, a study of this process would require a rather complete understanding of the collapse of the progenitor star within the full nonlinear theory of general relativity. The alternative is to study astrophysical systems involving neutron star oscillations that can be treated using perturbation theory. A system that allows such an approach and, at the same time, could be viewed as an approximation to a realistic astrophysical system is that of a "small" particle orbiting a neutron star. Here small is to be understood in the sense that the mass $\mu$ and size of the particle are much smaller than the mass $M$ and radius $R_{*}$ of the neutron star. Under this approximation, the particle moves along a geodesic in the spacetime generated by the neutron star, causing metric perturbations that propagate and eventually excite the neutron star. Of course, in principle those gravitational perturbations in turn would cause deviations of the particle's trajectory; however, these back-reaction effects are of second order and will therefore be neglected.

The specific goal of this paper is to investigate the gravitational radiation emitted from the orbiting and scattering of a particle by a neutron star. Collisions of particles with the neutron star [9] are not considered because it is not clear at all how to treat the impact and subsequent merge of the particle with the neutron star. Contrary to previous studies of excitations of neutron stars by orbiting particles, we perform our calculations in the time domain instead of the frequency domain 10 12]. In particular, we use the linearized ADM-formalism [13], which is naturally adapted for the numerical initial value problem. The relevant equations have been derived in [6], which we shall refer to as paper I. Considering the dynamics in the time domain has as a consequence that we are forced to "smooth out" the particle; that is, the $\delta$-functions in the sources of the equations due to the presence of the particle are approximated 
by Gaussians. We show, however, that our model of the particle is self-consistent and convergent. Another important issue is the prescription of appropriate initial data that satisfy the constraints.

The paper is organized as follows. In section II, we present the equations to describe the unperturbed configuration and its perturbations, as well as the method to treat the orbiting particle as a perturbation of the background spacetime. Section III deals with the numerical approximation of the particle terms in the equations. In section IV, we outline the problems with choosing appropriate initial data, and, by referring to the flat space problem, we give an approximate prescription of "good" initial data. In sections $\mathrm{V}$ and VI, we present and discuss our numerical results. Details concerning the derivation the particle terms can be found in the appendix.

\section{FORMULATION OF THE PROBLEM}

The energy-momentum tensor $\mathcal{T}^{\mu \nu}$ of a point particle with mass $\mu$ is given by

$$
\begin{aligned}
\mathcal{T}^{\mu \nu} & =\mu \int \delta^{(4)}\left(x^{\kappa}-X^{\kappa}(\tau)\right) u^{\mu} u^{\nu} d \tau \\
& =\mu \frac{U^{\mu} U^{\nu}}{U^{t} r^{2}} \delta(r-R(t)) \delta(\phi-\Phi(t)) \delta(\cos \theta-\cos \Theta(t)),
\end{aligned}
$$

where $U^{\mu}$ is the 4 -velocity of the particle

$$
U^{\mu}=\frac{d X^{\mu}}{d \tau}
$$

and $\tau$ the particle's proper time along its trajectory $X^{\kappa}(\tau)$.

We now orient the coordinate system in such a way that the particle's orbit coincides with the equatorial plain of the neutron star $(\Theta=\pi / 2)$. Besides, the particle's coordinate time $T$ is identical with the time coordinate $t$ of the spacetime in which it moves. Therefore we will use $t$ to parametrize the path of the particle $X^{\mu}(t)=$ $[t, R(t), \pi / 2, \Phi(t)]$.

From the geodesic equations $\frac{D U^{\mu}}{d \tau} \equiv U^{\nu} U_{; \nu}^{\mu}=0$ we find:

$$
\begin{aligned}
& \frac{d t}{d \tau}=e^{2 \lambda} E \\
& \frac{d R}{d \tau}=\left[E^{2}-e^{2 \nu}\left(1+\frac{L^{2}}{R^{2}}\right)\right]^{1 / 2} \\
& \frac{d \Phi}{d \tau}=\frac{L}{R^{2}}
\end{aligned}
$$

where $E$ and $L$ are the energy and angular momentum per unit mass of the particle, respectively. We also recall that for the Schwarzschild metric we have

$$
e^{2 \nu}=e^{-2 \lambda}=1-\frac{2 M}{r} .
$$

We can use (3a) to eliminate the proper time $\tau$ from equations $(3 \mathrm{~b})$ and $(3 \mathrm{c})$ :

$$
\begin{aligned}
& \frac{d R}{d t}=e^{2 \nu}\left[1-\frac{e^{2 \nu}}{E^{2}}\left(1+\frac{L^{2}}{R^{2}}\right)\right]^{1 / 2} \\
& \frac{d \Phi}{d t}=e^{2 \nu} \frac{L}{R^{2} E}
\end{aligned}
$$

Eqs. (5a) and (5b) can be used to replace the quantities $v_{r} \equiv \frac{d R}{d t}$ and $v_{\phi} \equiv \frac{d \Phi}{d t}$ in the source terms of the particle. However, we also have to explicitly solve them for the particle's trajectory coordinates $R(t)$ and $\Phi(t)$, since we need those coordinates in the $\delta$-functions $\delta(r-R(t))$ and $\delta(\phi-\Phi(t))$.

The background spacetime is solely due to the neutron star, which we model as a non-rotating polytrope with $\Gamma=2$ and $\kappa=100 \mathrm{~km}^{2}$. Outside the star, the spacetime is given by the Schwarzschild metric. The equations governing the stellar oscillations are derived in paper I. After expansion in Regge-Wheeler tensor harmonics, these equations can be grouped into two families, one describing the axial perturbations and the other describing the polar perturbations. 
In the exterior region, we now have to incorporate the source terms due to the particle into the perturbation equations. For that purpose, the energy-momentum tensor of the particle has to be expanded in tensor harmonics. The derivation of this expansion is given in the Appendix. There is a slight inconvenience created by the fact that the perturbation equations are expanded using the Regge-Wheeler harmonics [14, which do not form an orthonormal set, whereas the energy-momentum tensor of the particle is expanded using the orthonormal harmonics given by Zerilli [15]. However, the conversion is straightforward.

Even though the main focus will be on the polar perturbations, we also present the axial equations since they are so simple. In the Regge-Wheeler gauge, the only non-vanishing axial perturbations of the shift vector and spatial metric are respectively given by

$$
\begin{aligned}
\left(\beta_{\theta}, \beta_{\phi}\right) & =e^{\nu-\lambda} K_{6}^{l m}\left(-\sin ^{-1} \theta \frac{\partial}{\partial \phi} Y_{l m}, \sin \theta \frac{\partial}{\partial \theta} Y_{l m}\right) \\
\left(h_{r \theta}, h_{r \phi}\right) & =e^{\lambda-\nu} V_{4}^{l m}\left(-\sin ^{-1} \theta \frac{\partial}{\partial \phi} Y_{l m}, \sin \theta \frac{\partial}{\partial \theta} Y_{l m}\right)
\end{aligned}
$$

Similarly, the extrinsic curvature perturbations read

$$
\begin{aligned}
\left(k_{r \theta}, k_{r \phi}\right) & =\frac{1}{2} e^{\lambda} K_{3}^{l m}\left(-\sin ^{-1} \theta \frac{\partial}{\partial \phi} Y_{l m}, \sin \theta \frac{\partial}{\partial \theta} Y_{l m}\right) \\
\left(\begin{array}{ll}
k_{\theta \theta} & k_{\theta \phi} \\
k_{\phi \theta} & k_{\phi \phi}
\end{array}\right) & =e^{-\lambda} K_{6}^{l m}\left(\begin{array}{cc}
-\sin ^{-1} \theta X_{l m} & \sin \theta W_{l m} \\
\sin \theta W_{l m} & \sin \theta X_{l m}
\end{array}\right),
\end{aligned}
$$

with $W_{l m}$ and $X_{l m}$ defined in Eqs. (A6) and (A5) of the Appendix. With the inclusion of the particle terms the evolution equations read (see Paper I)

$$
\begin{aligned}
\frac{\partial V_{4}}{\partial t} & =e^{4 \nu}\left[\frac{\partial K_{6}}{\partial r}+2\left(\nu^{\prime}-\frac{1}{r}\right) K_{6}\right]-e^{2 \nu} K_{3} \\
\frac{\partial K_{3}}{\partial t} & =\frac{l(l+1)-2}{r^{2}} V_{4}-16 \pi e^{2 \nu} t_{7} \\
\frac{\partial K_{6}}{\partial t} & =\frac{\partial V_{4}}{\partial r}-8 \pi t_{10} .
\end{aligned}
$$

The momentum constraint relates the extrinsic curvature coefficients to the particle's source term via

$$
\frac{\partial K_{3}}{\partial r}+\frac{2}{r} K_{3}-\frac{l(l+1)-2}{r^{2}} K_{6}=16 \pi e^{2 \lambda} t_{4} .
$$

For completeness, we also list the source terms of the particle:

$$
\begin{aligned}
t_{4} & =e^{2 \nu} \frac{\mu L}{r^{2} l(l+1)} \delta(r-R(t)) \frac{\partial}{\partial \Theta} Y_{l m}^{*}\left(\frac{\pi}{2}, \Phi(t)\right) \\
t_{7} & =e^{2 \lambda} \frac{\mu L}{r^{2} l(l+1)} v_{r} \delta(r-R(t)) \frac{\partial}{\partial \Theta} Y_{l m}^{*}\left(\frac{\pi}{2}, \Phi(t)\right) \\
t_{10} & =-e^{2 \nu} \frac{2 \mathrm{i} m \mu L^{2}}{r^{2} E l(l+1)(l-1)(l+2)} \delta(r-R(t)) \frac{\partial}{\partial \Theta} Y_{l m}^{*}\left(\frac{\pi}{2}, \Phi(t)\right) .
\end{aligned}
$$

For radial infall, $L=0$, and all source terms vanish. Hence, in this case the radiation is of pure even parity.

Let us now turn to the polar perturbations. Here, we have to use some caution in including the particle terms. From the ADM split, we see that the matter terms enter only in the equations for the extrinsic curvature coefficients $\widehat{K}_{i}$. In the Regge-Wheeler gauge, we are only left with equations for the coefficients $\widehat{K}_{1}, \widehat{K}_{2}$ and $\widehat{K}_{5}$. In paper I, we showed that we have to choose initial data with $\widehat{K}_{4}=0$. Without the particle, the evolution equations then guaranteed the vanishing of $\widehat{K}_{4}$ for all times. However, in the presence of the particle, this is not true anymore since $\widehat{K}_{4}$ satisfies the following evolution equation:

$$
\frac{\partial}{\partial t} \widehat{K}_{4}=-8 \pi t_{8}
$$


This means that during the evolution $\widehat{K}_{4}$ will be become nonzero even if it was set to zero initially. A non-vanishing $\widehat{K}_{4}$ implies nonzero metric components $\widehat{T}_{1}$ and $\widehat{V}_{3}$; this violates the Regge-Wheeler gauge condition. A solution is to choose a different lapse function perturbation $\alpha$. If we pick

$$
\alpha=-\frac{1}{2} e^{\nu}\left(\frac{T}{r}+r S+16 \pi t_{8}\right) Y_{l m}
$$

the last term exactly cancels the right-hand side of (15) and the vanishing of $\widehat{K}_{4}$ and therewith $\widehat{T}_{1}$ and $\widehat{V}_{3}$ is, again, guaranteed.

The remaining metric quantities are expanded in the same way as in paper I:

$$
\begin{aligned}
\beta_{i} & =\left(e^{2 \lambda} K_{2}, 0,0\right) Y_{l m} \\
h_{i j} & =\left(\begin{array}{ccc}
e^{2 \lambda}\left(\frac{T}{r}+r S\right) & 0 & 0 \\
0 & r T & 0 \\
0 & 0 & r \sin ^{2} T
\end{array}\right) Y_{l m}
\end{aligned}
$$

and the expansion of the extrinsic curvature reads

$$
k_{i j}=-\frac{e^{-\nu}}{2 r}\left(\begin{array}{ccc}
e^{2 \lambda} K_{1} & -e^{2 \lambda} K_{2} \frac{\partial}{\partial \theta} & -e^{2 \lambda} K_{2} \frac{\partial}{\partial \phi} \\
\star & r^{2}\left(K_{5}-2 K_{2}\right) & 0 \\
\star & 0 & r^{2} \sin ^{2} \theta\left(K_{5}-2 K_{2}\right)
\end{array}\right) Y_{l m} .
$$

In paper I, we have shown that it is possible to write the evolution equations as a set of two coupled wave equations only in terms of the metric components $S$ and $T$. With the inclusion of the particle terms those evolution equations read in the exterior region

$$
\begin{aligned}
& \frac{\partial^{2} S}{\partial t^{2}}=e^{4 \nu}\left[\frac{\partial^{2} S}{\partial r^{2}}+6 \nu^{\prime} \frac{\partial S}{\partial r}+\left(4\left(\nu^{\prime}\right)^{2}-2 \frac{\nu^{\prime}}{r}-e^{2 \lambda} \frac{l(l+1)}{r^{2}}\right) S+4 \frac{\nu^{\prime}}{r^{2}}\left(\nu^{\prime}-\frac{3}{r}\right) T+\frac{16 \pi}{r} P_{1}\right] \\
& \frac{\partial^{2} T}{\partial t^{2}}=e^{4 \nu}\left[\frac{\partial^{2} T}{\partial r^{2}}+2 \nu^{\prime} \frac{\partial T}{\partial r}+\left(2 \frac{\nu^{\prime}}{r}-e^{2 \lambda} \frac{l(l+1)}{r^{2}}\right) T-2 S+16 \pi P_{2}\right],
\end{aligned}
$$

where the $P_{1}$ and $P_{2}$ denote the source terms of the particle

$$
\begin{aligned}
& P_{1}=t_{8}^{\prime \prime}-2 t_{6}^{\prime}+\left(5 \nu^{\prime}-\frac{3}{r}\right) t_{8}^{\prime}+t_{5}-2\left(3 \nu^{\prime}-\frac{1}{r}\right) t_{6}+2\left(\nu^{\prime}\left(\nu^{\prime}-\frac{6}{r}\right)+\frac{2}{r^{2}}\right) t_{8}-\frac{e^{2 \lambda}}{r^{2}} t_{9} \\
& P_{2}=t_{8}^{\prime}-2 t_{6}+2\left(\nu^{\prime}-\frac{1}{r}\right) t_{8}+\frac{e^{2 \lambda}}{r} t_{9}-\frac{r}{2} e^{2 \lambda} t
\end{aligned}
$$

with the $t_{i}$ given in the Appendix. The evolution equations which govern the oscillations in the interior of the neutron star are given in paper I (e.g. (49) and (58)). Furthermore, the perturbations in the exterior are completely described by the Zerilli equation (eqs. (61) of paper I):

$$
\frac{\partial^{2} Z}{\partial t^{2}}=\frac{\partial^{2} Z}{\partial r_{*}^{2}}+V(r) Z+S_{Z}
$$

with the following explicit form of the source term

$$
\begin{aligned}
S_{Z}=-16 \pi e^{4 \nu} \frac{\mu}{r^{2} E \Lambda(n+1)}[ & e^{2 \nu}\left(L^{2}+r^{2}\right) \delta^{\prime}(r-R)+\left(3 M\left(1+4 \frac{E^{2}}{\Lambda}\right)-r(n+1)-2 \mathrm{i} m e^{2 \mu} v_{r} L E\right. \\
& \left.\left.+\frac{L^{2}}{r^{2} n}\left(r n\left(m^{2}-3-2 n\right)-M\left(3-2 n-3 m^{2}\right)\right)\right) \delta(r-R)\right] Y_{l m}^{*},
\end{aligned}
$$

where $2 n=l(l+1)-2$. In addition, we have three constraint equations, namely the Hamiltonian constraint, which now reads

$$
T^{\prime \prime}+\nu^{\prime} T^{\prime}-r S^{\prime}-\left(5 \frac{\nu^{\prime}}{r}+e^{2 \lambda} \frac{l(l+1)}{r^{2}}\right) T-\left(2 r \nu^{\prime}+2+\frac{1}{2} e^{2 \lambda} l(l+1)\right) S=-8 \pi e^{2 \lambda} \frac{\mu E}{r} \delta(r-R(t)) Y_{l m}^{*},
$$


and two momentum constraints

$$
\begin{aligned}
r K_{5}^{\prime}-\frac{1}{2} e^{2 \lambda} l(l+1) K_{2}-r^{2} K-\left(r \nu^{\prime}+1\right) K_{5} & =8 \pi e^{2 \mu} \mu E v_{r} \delta(r-R(t)) Y_{l m}^{*} \\
r K_{2}^{\prime}-r^{2} K-2 K_{5} & =16 \pi e^{2 \nu} \frac{\mathrm{i} m \mu L}{r l(l+1)} \delta(r-R(t)) Y_{l m}^{*}
\end{aligned}
$$

Notice that the quantity $K_{2}$ appears in Eqs. (27) and (28) but not in the evolution equations. Therefore, we will eliminate it by differentiating (27) with respect to $r$ and using (27) and (28) to eliminate $K_{2}$ and $K_{2}^{\prime}$. The resulting equation is then second order in $K_{5}$ and reads

$$
\begin{aligned}
K_{5}^{\prime \prime}+\nu^{\prime} K_{5}^{\prime}-r K^{\prime} & -\left(5 \frac{\nu^{\prime}}{r}+e^{2 \lambda} \frac{l(l+1)}{r^{2}}\right) K_{5}-\left(2 r \nu^{\prime}+2+\frac{1}{2} e^{2 \lambda} l(l+1)\right) K \\
& =8 \pi \frac{\mu}{r}\left(e^{2 \lambda} v_{r} E \delta^{\prime}(r-R(t))+\mathrm{i} m L \delta(r-R(t))\right) Y_{l m}^{*} .
\end{aligned}
$$

It should be noted, that this equation also follows from taking the time derivative of the Hamiltonian constraint (26) and taking into account that $\dot{S}=K$ and $\dot{T}=K_{5}$.

As usual, the initial data must satisfy the constraint equations. Unfortunately, there is no unique way to solve those equations. This is due to the fact that to a particular solution of the inhomogeneous equations, we can always add a solution of the homogeneous equation, which would correspond to adding some arbitrary gravitational waves. The problem of finding the "right" initial data that represent only the perturbations which are due to the presence of the particle and which do not contain any additional radiation will be discussed in more detail in section IV.

For radial infall of a particle from rest, $v_{r}(t=0)=0$ and $L=0$, and therefore $t_{2}=t_{3}=0$. Thus the momentum constraints (27) and (28) can be trivially satisfied by setting the extrinsic curvature variables to zero. This situation corresponds to time symmetric initial data. We then are left with solving the Hamiltonian constraint (26). Of course, a particle falling from rest would fall radially towards the neutron star and eventually hit its surface. Since we want to avoid such an impact, we have to give the particle some angular momentum. In addition, we want to consider different initial radial velocities which means that we have to solve also the momentum constraints (27) and (28).

\section{NUMERICAL IMPLEMENTATION OF THE PARTICLE}

The presence of a particle in the calculations introduces source terms in the equations that require the explicit forms of the spherical harmonics $Y_{l m}$. The perturbation equations without particle are degenerate with respect to $m$ since the background metric is spherically symmetric. However, the particle breaks this symmetry, and one is forced to consider the various $m$-cases. Fortunately, we do not have to consider all possible values of $m$ for a given values of $l$ since for negative $m$ the spherical harmonics just undergo sign change and phase shift $\left(Y_{l m}^{*}=(-1)^{m} Y_{l,-m}\right)$. The advantage of putting the particle in the equatorial plane $\left(\Theta=\frac{\pi}{2}\right)$ is that, in the even parity case, we only have to deal with multipoles with $m=l, l-2, \ldots$, the remaining ones $(m=l-1, l-3, \ldots)$ have odd parity. Since the evolution code handles only real valued perturbations, we have to treat the real and imaginary parts of the spherical harmonics separately. Finally, all the equations will be solved on a finite grid, hence we have to approximate the $\delta$-function by a narrow Gaussian

$$
\delta(r-R(t)) \approx \frac{1}{\sigma \sqrt{2 \pi}} e^{-\frac{(r-R(t))^{2}}{2 \sigma^{2}}} \quad \sigma \text { small } .
$$

To demonstrate the validity of this approximation, we have to ensure the convergence of the solution for $\sigma \rightarrow 0$. This can be done in two different ways. One can look at the convergence of the waveforms that are obtained in the evolution, or also one can monitor the violation of the constraints. A possible way do to so is to monitor the following quantity

$$
I=\frac{1}{8 \pi \mu E Y_{l m}^{*}} \int r e^{2 \nu}(\text { lhs of } 26 \text { ) }) d r,
$$

where the domain of integration is the region outside the neutron star. In the limit $\sigma \rightarrow 0$, one gets that $I=1$ throughout the whole evolution. Numerically, we cannot take this limit with a fixed grid size since, eventually, we cannot sufficiently resolve the Gaussian. To check the convergence, we decrease both $\sigma$ and $\Delta x$ by keeping the ratio $\sigma / \Delta x$ constant throughout the sequence. By consecutively doubling the resolution and halving $\sigma$, we find that $I-1$ 
approaches zero with second order convergence. For the numerical evolutions, we will use a value of $\sigma / \Delta x=0.15$, which gives provides a suitable resolution of the Gaussian and its derivatives.

There is still a subtle point. In deriving the source terms (see Appendix), we have tacitly transformed the particle coordinate $R$ into the spacetime coordinate $r$ since the presence of the $\delta$-function makes a distinction unnecessary. However, in the evolution equations we have to take derivatives of the source terms with respect to $r$ but not with respect to $R$, and therefore we would obtain different source terms if we had not changed the $R$ 's into $r$ 's. As an example consider the following two source terms

$$
S_{1}(r)=f(r) \delta(r-R)
$$

and

$$
S_{2}(r)=f(R) \delta(r-R),
$$

which are equivalent because of the presence of the $\delta$-function. But if we now differentiate $S_{1}$ and $S_{2}$ with respect to $r$ we obtain for $S_{2}$ just the derivative of the $\delta$-function, whereas for $S_{1}$ we also have to differentiate $f$. Analytically this does not make a difference, but if we approximate the $\delta$-function by a Gaussian, then the two expressions for $S_{1}$ and $S_{2}$ and their respective derivatives are different. To gain accuracy, we should have kept $r^{2} d \phi / d \tau \delta(r-R)$ as $L r^{2} / R^{2} \delta(r-R)$ and not just as $L \delta(r-R)$. However, for the numerical evolutions the actual difference is negligible, so we have assumed the source terms to be of the form of $S_{1}$.

\section{SETTING UP THE INITIAL CONDITIONS}

Any construction of initial data for a particle initially located at $\left(r_{0}, \Phi_{0}\right)$ with initial radial velocity $v_{r}$ and angular momentum $L$ involves solving the Hamiltonian constraint (26). Usually, we set the initial angle $\Phi_{0}$ to zero. However, Eq. (26) contains two quantities $S$ and $T$. This means that there is some freedom in choosing the initial values. We can e.g. either set the quantity $T$ to zero and solve for $S$, or do it the other way round and set $S=0$ and solve for $T$. In the former case, we would have to solve a first order equation for $S$, and the latter case would lead to a second order equation for $T$. In Fig. 1 we show both possible sets of initial data.

To assess which choice is the more natural, we consider a particle initially at rest in flat spacetime. Of course, the particle will remain at rest since there is no matter around that could attract the particle. Thus, the perturbation of the spacetime that is created by the particle will be stationary. Hence the equations of motion for the metric perturbations $S$ and $T$ will read $\frac{\partial S}{\partial t}=0$ and $\frac{\partial T}{\partial t}=0$. From these conditions and from the Hamiltonian constraint, it then follows that $S$ has to vanish.

Of course, in the presence of the neutron star, those arguments do not hold any more, and $S \equiv 0$ will not be the right choice of initial data, but if the particle is initially far enough away from the neutron star, the error in setting $S=0$ should be very small. This error actually corresponds to an introduction of an extra amount of gravitational radiation that is not at all related to the radiation that is emitted when the particle moves through the spacetime. This extra amount will start to propagate during the evolution and eventually hit the neutron star and thereby cause it to oscillate. However, if we put the particle far enough away from the neutron star, the strength of the induced oscillation should be small compared to the ones excited when the particle comes close to the neutron star.

In Figs. 2 and 3, we show the evolution of the two possible choices of initial data for a particle falling from rest. The left panels show the evolution of $S$ and $T$ in the case $T_{0}=0$, whereas the right panels show the evolution of $S$ and $T$ with $S_{0}=0$. The differences are obvious. The initial shape of $T$ in the latter case is almost unchanged during the evolution, whereas $S$ starts to acquire its "right" shape. In the other case, we can see a huge burst of radiation propagating in both directions. In the same time $T$ is acquiring its "right" shape. In Fig. 4, we show the evolution of the Hamiltonian constraint where we evaluate the left-hand side of (26) which monitors the "path" of the particle. In both cases the graphs agree. Lastly, in Fig. 5, we also show the metric functions $S$ and $T$ at the end of the evolution. This figure clearly show that, regardless of the chosen initial data, the functions $S$ and $T$ will adjust to their proper values after having radiated away the superfluous initial wave content.

We should note that we cannot escape the whole ambiguity of how to choose the variables $S$ and $T$ by using the Zerilli formalism, instead. There, any regular initial data are valid initial data since by construction the Zerilli function always satisfies the constraint equations. Hence, we cannot see a priori whether or not the chosen initial data will have additional radiation content.

If the particle is initially not at rest, in addition to solving the Hamiltonian constraint (26), we also have to solve the momentum constraints (27) and (28). Of course, as with the Hamiltonian constraint, we are again faced with the same kind of ambiguities in solving that equation. 
Now, for a particle being initially at rest or very slow, or for circular orbits, we do not worry about what kind of initial data we choose. Since any initial gravitational wave pulse travels with the speed of light and is therefore much faster than the particle, it will long be gone when the particle comes close to the star. However, if the particle's initial velocity is close to the speed of light, then the particle "rides" on its own wave pulse, and it will be not clear any more whether the excitation of some particular modes of the neutron star is due the the particle itself or due to the initial burst which comes from inappropriate initial data. If the particle is slow enough, those two effects can clearly be distinguished. For very fast particles, this is not possible any more. This is particularly bothersome given that a pulse of gravitational waves will predominately excite $w$-modes. If we have a wave signal from a particle that grazes a neutron star with almost the speed of light, and we find that, indeed, there are some traces of a $w$-mode, it would be very difficult to state that the excitation of $w$-modes is a "real" signal and not an artifact due to the inappropriate initial data.

To obtain an approximate answer, we again turn to the flat space case. In this limit, the equations governing the evolution of $S$ and $T$ reduce to two simple coupled wave equations with a source term for each that takes into account the presence of the particle:

$$
\begin{aligned}
& \frac{\partial^{2} S}{\partial t^{2}}=\frac{\partial^{2} S}{\partial r^{2}}-\frac{l(l+1)}{r^{2}} S+16 \pi \mu E \frac{v^{2}}{r^{3}} \delta(r-R(t)) Y_{l m}^{*} \\
& \frac{\partial^{2} T}{\partial t^{2}}=\frac{\partial^{2} T}{\partial r^{2}}-\frac{l(l+1)}{r^{2}} T+4 S-8 \pi \frac{\mu}{r E} \delta(r-R(t)) Y_{l m}^{*}
\end{aligned}
$$

Herein, we have omitted the terms proportional to $L$, since we let the particle move on a radial trajectory with constant velocity $v$, hence it is $L=0$ and

$$
R(t)=R_{0}+v t
$$

with $R_{0}$ being the initial location of the particle. Furthermore, the normalized energy $E$ of the particle is just given by the Lorentz factor

$$
E=\frac{1}{\sqrt{1-v^{2}}}
$$

It is interesting to note that the wave equation for $S$ is totally decoupled from the one for $T$. However the solutions of (34) and (35) have to satisfy the flat space Hamiltonian constraint which reads

$$
\frac{\partial^{2} T}{\partial r^{2}}-\frac{l(l+1)}{r^{2}} T-r \frac{\partial S}{\partial r}-\left(2+\frac{1}{2} l(l+1)\right) S=-8 \pi \frac{\mu E}{r} \delta(r-R(t)) Y_{l m}^{*} .
$$

We now seek an exact solution of (34) that obeys the right boundary conditions at the origin and at infinity. Once found, we may use (38) to numerically compute the appropriate $T$. We state that a solution for (34) is given by the following series ansatz:

$$
S(t, r)=A\left(\sum_{i=0} a_{i} \frac{r^{2 i+l+1}}{\left(R_{0}+v t\right)^{2 i+l+3}} \Theta\left(R_{0}+v t-r\right)+\sum_{i=0} b_{i} \frac{\left(R_{0}+v t\right)^{l-2 i-2}}{r^{l-2 i}} \Theta\left(r-R_{0}-v t\right)\right)
$$

where $\Theta$ is the Heaviside function which satisfies

$$
\Theta(x)= \begin{cases}0, & x<0 \\ 1, & x \geq 0\end{cases}
$$

Continuity at $r=R_{0}+v t$ requires that

$$
\sum_{i=0} a_{i}=\sum_{i=0} b_{i}
$$

The overall amplitude will be determined by $A$, hence we deliberately may set

$$
\sum_{i=0} a_{i}=\sum_{i=0} b_{i}=1 .
$$


The amplitude $A$ and the coefficients $a_{i}$ and $b_{i}$ can be found by plugging (39) into (34). For $A$ we find

$$
A=\frac{16 \pi \mu E^{3} v^{2} Y_{l m}^{*}}{2 l+1+2 \sum_{i=0} i\left(a_{i}-b_{i}\right)}
$$

and the coefficients $a_{i}$ and $b_{i}$ are determined by the following recursion relations

$$
\begin{aligned}
a_{i+1} & =a_{i} v^{2} \frac{(2 i+l+3)(2 i+l+4)}{2(i+1)(2 i+2 l+3)} \\
b_{i+1} & =b_{i} v^{2} \frac{(l-2 i-2)(l-2 i-3)}{2(i+1)(2 i-2 l+1)}
\end{aligned}
$$

It is interesting to note that, while the series in $a_{i}$ never terminates, the series in $b_{i}$ always terminates because one of the two factors in the numerator will become zero for some $i$. The series in $a_{i}$ converges if and only if $|v|<1$.

In Fig. 6, we show the initial data obtained from (39) for a particle that is located at $R_{0}=500 \mathrm{~km}$ with different initial velocities. Here, instead of $S$ and $T$, we plot the quantities $r S$ and $T / r$, since from (18) it is clear that only those expressions allow direct comparisons. For $v=0$ it is $r S=0$ but the amplitude of $r S$ grows rapidly when the particle's velocity approaches the speed of light, whereas the peak of $T / r$ slightly decreases. In the ultra-relativistic limit $r S$ totally dominates over $T / r$.

The solutions above for $S$ and $T$ are not valid any more if we consider the particle in curved spacetime because they would violate the constraints. However, we can still use (39) as a prescription for $S$ and then use the curved space Hamiltonian constraint (26) to solve for $T$. Furthermore, we can compute $K$ from $K=d S / d t$ and then use the momentum constraint (29) to compute $K_{5}$. As long as the particle does not have any angular momentum and is far away from the neutron star, the thus obtained initial values should be a good approximation for a boosted particle on a Schwarzschild background. But if the particle has a large angular momentum, there will be additional source terms in the evolution equations and our approximation should break down. However, we are mainly interested in trajectories which come very close to the neutron star, and, hence, have only small angular momentum. In this case the above prescription still yields good initial data.

\section{NUMERICAL RESULTS}

To compare the code with known results, we first consider a particle in a circular orbit around the neutron star and compute the radiated energy at infinity. Numerically, this can be accomplished by evaluating the Zerilli function at some large distance. In terms of the metric variables $S$ and $T$, the Zerilli function reads

$$
Z=-\frac{2}{l(l+1)} \frac{1-\frac{2 M}{r}}{l(l+1)-2+\frac{6 M}{r}}\left(2 r T^{\prime}+\frac{2 M-r(2+l(l+1))}{r-2 M} T-2 r^{2} S\right) .
$$

The radiated power for some particular values of $l$ and $m$ can then be computed from

$$
\frac{d E_{l m}}{d t}=\frac{1}{64 \pi} \frac{(l+2) !}{(l-2) !}\left|\frac{Z_{l m}}{d t}\right|^{2} .
$$

Cutler et al. [16] have numerically computed the normalized gravitational power $(M / \mu)^{2} d E_{l m} / d t$ radiated by a particle orbiting a Schwarzschild black hole for various values of $l, m$ and $R_{0} / M$. In table II, they show the multipole components for $R_{0} / M=10$. To compare the output of our code to those results, we chose the mass of the neutron star to be $M=1.99 \mathrm{~km}$ and the orbit of the particle the be at $R_{0}=19.9 \mathrm{~km}$ in order to obtain the ratio of $R_{0} / M=10$. The mass of the particle was set to $\mu=1 \mathrm{~km}$. In Fig. 17. we show $d Z_{l m} / d t$ as a function of time extracted at $r=500 \mathrm{~km}$ for $l=m=2$. After some wave bursts that come from the inappropriate choice of initial data, we see that the signal is periodic with a frequency of twice the orbital frequency of the particle. The amplitude is about 0.0076 which corresponds to a radiated power of $(M / \mu)^{2} d E_{22} / d t=5.46 \times 10^{-5}$ which is in excellent agreement with Cutler et al. who obtain a value of $(M / \mu)^{2} d E_{22} / d t=5.388 \times 10^{-5}$. The slightly higher value of our result may be due to the fact that at $r=500 \mathrm{~km}$ the Zerilli function is still somewhat off its asymptotic value which will be a little bit smaller. And, of course, a neutron star is not a black hole, since its oscillation spectrum is quite different. But as long as the particle does not excite the eigenmodes in a significant way, i.e. as long as the particle's orbital frequency is not in resonance with one of the eigenmodes, the emitted radiation should be quite similar to the black hole case. And, indeed, we find that we agree with all the even and odd parity modes compiled in table II of Cutler et al. within a 
few percent, only for the case $l=3$ and $m=1$ we find the radiated power to be $5.9 \times 10^{-10}$ instead of their value of $5.71 \times 10^{-8}$.

If the orbital frequency is in resonance with one of the eigenmodes, the emitted radiation can drastically increase 17]. It is clear, however, that only the low-frequency modes such as the $f$-mode can be excited by this mechanism, the frequencies of the $w$-modes are much too high to be considerably excited. Besides, due to their strong damping, there is no sharp resonance for the $w$-modes at all, it is only for the weakly damped fluid modes that resonant mode excitation can occur.

Therefore, the only way to possibly excite $w$-modes are very eccentric orbits, where the periastron is very close to the surface of the star or scattering processes with very small impact parameters. The investigation of the latter is the main objective of this work. Before presenting the results, we should point out some problems that are related with the use of an evolution code.

Ideally, one should extract the waveform at $r=\infty$ which is clearly not possible on a finite grid. We have to record the waveform at some finite distance from the star. But this means that if the particle moves on an unbounded orbit it will eventually cross the location of the observer who will be located on a finite radius $r_{\text {obs }}$. Of course, the particle is always slower than the propagation speed of the gravitational waves, hence the observer will see the wave signal first before the particle crosses $r_{o b s}$. However, when the particle is very fast and the observer is not far enough away from the neutron star, then the signal and the particle will cross the observer almost at the same time. That means that the signal will be a superposition of the "real" gravitational wave signal and the influence of the gravitational field of the particle itself. The farther the observer moves outwards the better the separation of the two components in the signal can be made. Furthermore, the amplitude of the signal remains constant for increasing $r$, whereas the influence of the particle decreases at least as $1 / r$. The effectiveness of this undesired influence of the particle strongly depends on the actual excitation strength of the neutron star oscillations. Smaller turning points $r_{t}$ of the particle will induce oscillations with higher amplitudes and thus make the influence of the particle undetectable. For large $r_{t}$ the induced fluid oscillations are so weak that they will totally be buried within the gravitational field of the particle. Figure 8 drastically shows the influence of the presence of the particle on the observed waveform. Here, we compare the signals that result for two scattering orbits with $r_{t}=10 \mathrm{~km}$ and $r_{t}=50 \mathrm{~km}$. One can also see that the effect is much more pronounced in the waveform of $T$. But regardless of this unwanted feature it is still possible to infer qualitative statements from the obtained wave signals.

Unfortunately, the influence of the source terms is even much worse for the Zerilli function, which should be used to present the results, since it is a gauge invariant quantity and directly related to the radiated power. This is not the case for our variables $S$ and $T$. But since we are mainly interested in assessing which modes are excited by the scattering process, we do not have to compute the Zerilli function. It is enough to just examine the waveforms of $S$ and $T$.

In Figs. 9 through 11, we show the waveforms of $S$ and $T$ for different initial radial velocities $v_{0}$ and different values of the radius of the turning point $r_{t}$. In Figs. 12 through 14 we show the associated power spectra.

For all cases considered, the waveforms always have the same overall features. The first part of the signal consists of an increase in the amplitude that results of the particle entering the strong gravitational field of the neutron star. After a few irregular oscillations which correspond to the particle curving around the neutron star, the final signal will be dominated by the quasi-normal ringing of the neutron star, which is confirmed by Fourier analyzing the signal.

For slow particles the power spectrum shows one broad peak around the frequency that corresponds the the angular velocity of the particle at the turning point $r_{t}$ and some little sharp peaks that correspond to the fluid modes of the neutron star ( $f$-mode and lowest $p$-modes). From the waveforms it is clear that the excitations strengths of the modes decrease rapidly as the turning point $r_{t}$ of the particle moves farther away from the neutron star.

When the particle reaches the speed of light, the picture changes drastically. For initial velocities $v_{0} \lesssim-0.6$ the appearance of the $w$-mode becomes evident. In the ultra-relativistic case of $v_{0}=-0.97$ (Fig. 11) which corresponds to $E \approx 4.5$ it is clear that the lion's share of the energy gets radiated through the first $w$-mode channel. Note, that in obtaining the power spectrum of Fig. 14, we have cut off the initial radiation and have taken the Fourier transformation right where the $w$-mode ringing starts. It is interesting to see that even if the particle's turning point is quite far away $\left(r_{t}=50 \mathrm{~km}\right)$ the $w$-mode is still visible.

All the calculations have been performed using a stellar model with central energy density of $\epsilon_{0}=5.7 \times 10^{15} \mathrm{~g} \mathrm{~cm}^{-3}$ which leads to a mass of $1.35 \mathrm{M}_{\odot}$ and radius of $7.52 \mathrm{~km}$. This model is right below the maximum mass that is allowed for the polytropic equation of state with $\Gamma=2$ and $\kappa=100 \mathrm{~km}^{2}$. It is clear that the results will not change too much if we change the equation of state, or if we consider models with different masses. Only for very light neutron stars, the $w$-modes can become invisible again. For ultra-compact stars, we expect the long-lived trapped modes to be a dominant part of the power spectrum. 


\section{CONCLUSIONS}

We have performed simulations of particles on circular orbits as well as on scattering trajectories. The circular orbits were done for code testing reasons, and we found that the radiated energies agree perfectly with those obtained by Cutler et al. [16] who looked at a particle orbiting a black hole.

For the scattering orbit, we discussed the numerical difficulties associated with an evolution code. However, the results are accurate enough to address the issue of the excitation of $w$-modes by orbiting particles. First of all, it became clear that in order to excite a significant amount of $w$-modes the particle's velocity at infinity must be $\gg 0.5 \mathrm{c}$. Secondly, the excitation strength of the modes rapidly decreases as the particle's turning point $r_{t}$ increases. This is in agreement with the results of Ferrari et al. 10, who report that no significant $w$-mode excitation is observable. However, they did not consider the case where the particle has an energy $E \gg 1$, which is necessary if it were to excite $w$-modes. This was done by Andrade and Price for the odd-parity case 112]. Here, too, they find excitations of $w$-modes.

The following question now arises: Can we infer anything about what happens in an astrophysical event? Our results show that the particle's initial velocity has to be incredibly high in order to produce a significant amount of $w$-mode excitation. It is clear that there do not exist any astrophysical events at all that could accelerate the particle (which, of course, represents some heavy extended object, like a planet or even another neutron star) up to some significant fraction of the speed of light. Hence, we might safely conclude that any astrophysical scenario that might be simulated by a particle orbiting a neutron star will not produce any detectable amount of $w$-modes.

The alternative is then to excite $w$-modes from the collision of the particle with the neutron star. Our method could not be applied to this situation, but we could speculate about the possible implications. It has been suggested that in this case there might be some significant excitation of $w$-modes. In particular, simulations of a particle that spirals onto a ultra-compact constant density star where done by Borelli [9] who found that, indeed, both the trapped modes and the $w$-modes where excited, the latter much less, however, than the former. One serious drawback of those calculations, however, was that as soon as the particle had hit the surface of the star, the calculation was stopped. This lead to an overestimation of the high frequency components in the power spectrum which, of course, are represented by the $w$-modes. Since in this study, only odd parity modes were investigated, it is clear that the $w$-modes will always show up in the spectrum because they will not be screened by the presence of fluid modes what can happen in the even parity case. Now, even in the particle limit, it is not possible to simulate a collision since it not clear at all what happens if the particle hits the surface.

A first step, though, might be to let the particle go right through the neutron star without being affected by the presence of the neutron star matter. If this creates strong $w$-mode excitations even for particles with low initial velocities, then one could conclude that, in a realistic scenario where the physics of the impact is included, there still might be $w$-modes present. Of course, in this case, it seems reasonable to infer that at least the fluid modes would be much more strongly excited than in a scattering event.

Furthermore, our results seem to indicate that the presence of the $w$-modes in the signal is somehow related with the value of $S$. We have seen that for ultra-relativistic particles the values of $r S$ greatly dominates the one of $T / r$. And it is only there where we find a significant amount of $w$-modes. This particular role of $S$ seems to be corroborated by the results of paper I, where it was shown that the occurrence of $w$-modes strongly depends on the chosen initial data. It was found that for conformally flat initial metric perturbations (i.e. $S_{0}=0$ ) the evolution shows practically no $w$-modes at all. However, if one takes the same initial fluid perturbation, sets $T_{0}=0$ and solves for $S$, suddenly one can observe a quite large excitation of the $w$-modes in the signal. This also agrees with results in [5] and [7]

Still, the question remains, as to what happens in a realistic scenario. We have excluded the possibility of $w$ modes excitation by means of a realistic scattering process. However, it is not possible to relate our results to the merger process of a binary neutron star system since one cannot adequately described the system within perturbation theory. Only, if the final object does not immediately collapse to a black hole, it might wildly oscillate and emit some significant radiation through $w$-modes. It will only be through nonlinear evolutions that this issue might finally be settled.

\section{ACKNOWLEDGMENTS}

Work supported in part by NSF grants PHY9800973 and PHY9800970. J.R. was supported by a HSPIII grant from the DAAD (Germany). 


\section{APPENDIX A: THE SOURCE TERMS OF THE PARTICLE}

The particle's energy-momentum tensor (1) will be expanded using the orthonormal set of tensor harmonics $\left\{\left[\widehat{\mathcal{Y}}_{l m}^{A}\right]_{\mu \nu}\right\}_{A=1 . .10}$ given by Zerilli [15]:

$$
\mathcal{T}_{\mu \nu}=\sum_{l=0}^{\infty} \sum_{m=-l}^{l} \sum_{A=1}^{10} \hat{t}_{A}^{l m}\left[\widehat{\mathcal{Y}}_{l m}^{A}\right]_{\mu \nu}
$$

The coefficients $\hat{t}_{A}^{l m}$ can be obtained by means of the orthogonality relation

$$
\int_{S^{2}}\left[\widehat{\mathcal{Y}}_{l m}^{A}\right]^{* \mu \nu}\left[\widehat{\mathcal{Y}}_{l^{\prime} m^{\prime}}^{A^{\prime}}\right]_{\mu \nu} \mathrm{d} \Omega=s_{A} \delta_{A A^{\prime}} \delta_{l l^{\prime}} \delta_{m m^{\prime}},
$$

where the asterisk denotes complex conjugation and

$$
\left[\widehat{\mathcal{Y}}_{l m}^{A}\right]^{\mu \nu}=\eta^{\mu \kappa} \eta^{\nu \sigma}\left[\widehat{\mathcal{Y}}_{l m}^{A}\right]_{\kappa \sigma}
$$

Because of the use of the inverse Minkowsky metric $\eta^{\mu \nu}$ to raise the indices, the inner product (A2) is not positive definite and it is $s_{A}=-1$ for the harmonics with nonzero $0 j$-components $(A=2,3,4)$.

By using the orthonormality condition (A2) we can compute the coefficients $\hat{t}_{A}^{l m}$ through

$$
\hat{t}_{A}^{l m}=\int_{S^{2}}\left[\widehat{\mathcal{Y}}_{l m}^{A}\right]^{* \mu \nu} \mathcal{T}_{\mu \nu} \mathrm{d} \Omega
$$

We thus obtain the following set ( [18] with some corrections):

$$
\begin{aligned}
& {[t t] \quad \hat{t}_{1}^{l m}=e^{4 \nu} \frac{\mu}{r^{2}} \frac{d t}{d \tau} \delta(r-R(t)) Y_{l m}^{*}} \\
& {[t r] \quad \hat{t}_{2}^{l m}=\mathrm{i} \sqrt{2} \frac{\mu}{r^{2}} \frac{d R}{d \tau} \delta(r-R(t)) Y_{l m}^{*}} \\
& {\left[\begin{array}{c}
t \theta \\
t \phi
\end{array}\right]\left\{\begin{array}{l}
\hat{t}_{3}^{l m}=e^{2 \nu} \frac{2 \mathrm{i} \mu}{r \sqrt{2 l(l+1)}} \delta(r-R(t)) \frac{d}{d \tau} Y_{l m}^{*} \\
\hat{t}_{4}^{l m}=e^{2 \nu} \frac{2 \mu}{r \sqrt{2 l(l+1)}} \delta(r-R(t))\left(\frac{1}{\sin \Theta} \frac{d \Theta}{d \tau} \frac{\partial}{\partial \Phi}-\sin \Theta \frac{d \Phi}{d \tau} \frac{\partial}{\partial \Theta}\right) Y_{l m}^{*}
\end{array}\right.} \\
& {[r r] \quad \hat{t}_{5}^{l m}=e^{4 \lambda} \frac{\mu}{r^{2}} \frac{d \tau}{d t}\left(\frac{d R}{d \tau}\right)^{2} \delta(r-R(t)) Y_{l m}^{*}} \\
& {\left[\begin{array}{c}
r \theta \\
r \phi
\end{array}\right]\left\{\begin{array}{l}
\hat{t}_{6}^{l m}=e^{2 \lambda} \frac{2 \mu}{r \sqrt{2 l(l+1)}} \frac{d \tau}{d t} \frac{d R}{d \tau} \delta(r-R(t)) \frac{d}{d \tau} Y_{l m}^{*} \\
\hat{t}_{7}^{l m}=e^{2 \lambda} \frac{2 \mathrm{i} \mu}{r \sqrt{2 l(l+1)}} \frac{d \tau}{d t} \frac{d R}{d \tau} \delta(r-R(t))\left(\sin \Theta \frac{d \Phi}{d \tau} \frac{\partial}{\partial \Theta}-\frac{1}{\sin \Theta} \frac{d \Theta}{d \tau} \frac{\partial}{\partial \Phi}\right) Y_{l m}^{*}
\end{array}\right.}
\end{aligned}
$$

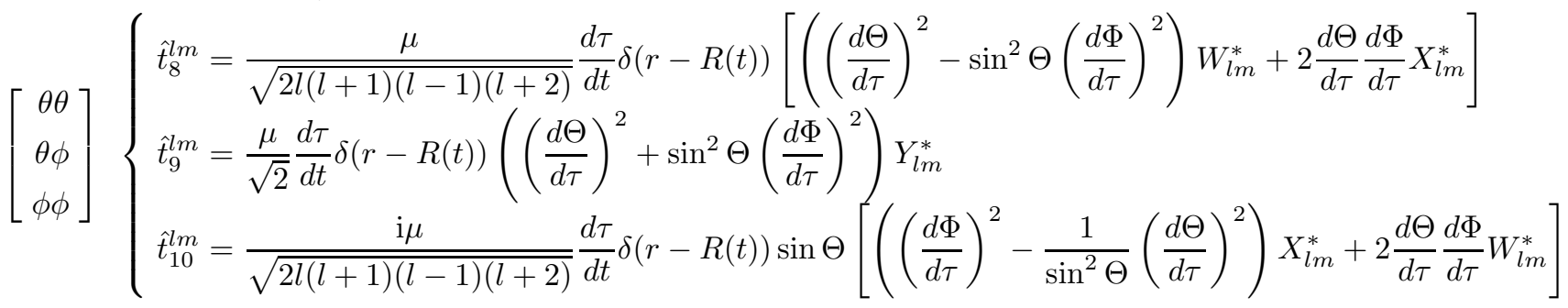

with

$$
\begin{aligned}
X_{l m} & :=2\left(\frac{\partial}{\partial \theta}-\cot \theta\right) \frac{\partial}{\partial \phi} Y_{l m} \\
W_{l m} & :=\left(l(l+1)+2 \frac{\partial^{2}}{\partial \theta^{2}}\right) Y_{l m} .
\end{aligned}
$$


Here, $Y_{l m}^{*}, W_{l m}^{*}$ and $X_{l m}^{*}$ are functions of the particle's angular position $\Theta$ and $\Phi$ parametrized by the coordinate time $t$, therefore all derivatives with respect to proper time $\tau$ are to be understood as

$$
\frac{d}{d \tau}=\frac{d t}{d \tau} \frac{d}{d t}=e^{2 \lambda} E \frac{d}{d t} .
$$

Since the evolution equations are expanded using the Regge-Wheeler harmonics, which do not form an orthonormal set, we have to convert the above coefficients $\hat{t}_{A}^{l m}$ into the coefficients $t_{A}^{l m}$ that would result from an expansion of the energy-momentum tensor into Regge-Wheeler harmonics. Using the relationship between the different sets of tensor harmonics given in [15] and [19] we find:

$$
\begin{aligned}
t_{1}^{l m} & =\hat{t}_{1}^{l m} \\
t_{2}^{l m} & =\frac{\mathrm{i}}{\sqrt{2}} \hat{t}_{2}^{l m} \\
t_{3}^{l m} & =\frac{\mathrm{i} r}{\sqrt{2 l(l+1)}} \hat{t}_{3}^{l m} \\
t_{4}^{l m} & =-\frac{r}{\sqrt{2 l(l+1)}} \hat{t}_{4}^{l m} \\
t_{5}^{l m} & =\hat{t}_{5}^{l m} \\
t_{6}^{l m} & =\frac{r}{\sqrt{2 l(l+1)}} \hat{t}_{6}^{l m} \\
t_{7}^{l m} & =-\frac{\mathrm{i} r}{\sqrt{2 l(l+1)}} \hat{t}_{7}^{l m} \\
t_{8}^{l m} & =\frac{2 r^{2}}{\sqrt{2 l(l+1)(l-1)(l+2)}} \hat{t}_{8}^{l m} \\
t_{9}^{l m} & =\frac{r^{2}}{\sqrt{2}}\left(\hat{t}_{9}^{l m}+\sqrt{\frac{l(l+1)}{(l-1)(l+2)}} \hat{t}_{8}^{l m}\right) \\
t_{10}^{l m} & =-\frac{2 \mathrm{i} r^{2}}{\sqrt{2 l(l+1)(l-1)(l+2)}} \hat{t}_{10}^{l m} .
\end{aligned}
$$

We now restrict the motion of the particle to the equatorial plane $\Theta=\frac{\pi}{2}$. In this case it is $\frac{d \Theta}{d \tau}=0$ and $\sin \Theta=1$, and we can use the geodesic equations (3a) and (3c) to substitute all expressions containing derivatives with respect to proper time $\tau$. Furthermore, we can obtain quite simple relations for the derivatives of $Y_{l m}^{*}$ :

$$
\begin{aligned}
\frac{\partial}{\partial \Phi} Y_{l m}^{*} & =-\mathrm{i} m Y_{l m}^{*} \\
\frac{\partial^{2}}{\partial \Theta^{2}} Y_{l m}^{*} & =\left(m^{2}-l(l+1)\right) Y_{l m}^{*} .
\end{aligned}
$$


This gives us a somewhat simpler set of coefficients:

$$
\begin{aligned}
t_{1}^{l m} & =e^{2 \nu} \frac{\mu E}{r^{2}} \delta(r-R(t)) Y_{l m}^{*} \\
t_{2}^{l m} & =-e^{2 \lambda} \frac{\mu E}{r^{2}} v_{r} \delta(r-R(t)) Y_{l m}^{*} \\
t_{3}^{l m} & =e^{2 \nu} \frac{\mathrm{i} m \mu L}{r^{2} l(l+1)} \delta(r-R(t)) Y_{l m}^{*} \\
t_{4}^{l m} & =e^{2 \nu} \frac{\mu L}{r^{2} l(l+1)} \delta(r-R(t)) \frac{\partial}{\partial \Theta} Y_{l m}^{*} \\
t_{5}^{l m} & =e^{6 \lambda} \frac{\mu E}{r^{2}} v_{r}^{2} \delta(r-R(t)) Y_{l m}^{*} \\
t_{6}^{l m} & =-e^{2 \lambda} \frac{\mathrm{i} m \mu L}{r^{2} l(l+1)} v_{r} \delta(r-R(t)) Y_{l m}^{*} \\
t_{7}^{l m} & =e^{2 \lambda} \frac{\mu L}{r^{2} l(l+1)} v_{r} \delta(r-R(t)) \frac{\partial}{\partial \Theta} Y_{l m}^{*} \\
t_{8}^{l m} & =e^{2 \nu} \frac{\mu L^{2}\left(l(l+1)-2 m^{2}\right)}{r^{2} E l(l+1)(l-1)(l+2)} \delta(r-R(t)) Y_{l m}^{*} \\
t_{9}^{l m} & =e^{2 \nu} \frac{\mu L^{2}\left(l(l+1)-m^{2}-1\right)}{r^{2} E(l-1)(l+2)} \delta(r-R(t)) Y_{l m}^{*} \\
t_{10}^{l m} & =-e^{2 \nu} \frac{2 \mathrm{i} m \mu L^{2}}{r^{2} E l(l+1)(l-1)(l+2)} \delta(r-R(t)) \frac{\partial}{\partial \Theta} Y_{l m}^{*},
\end{aligned}
$$

where $v_{r}=\frac{d R}{d t}$ is the radial velocity of the particle. The field equations also require the computation of the trace

$$
\mathcal{T}=g^{\mu \nu} \mathcal{T}_{\mu \nu}=\sum_{l, m} t^{l m} Y_{l m}
$$

with

$$
t^{l m}=-e^{2 \lambda} t_{1}^{l m}+e^{-2 \lambda} t_{5}^{l m}-\frac{l(l+1)}{r^{2}} t_{8}^{l m}+\frac{2}{r^{2}} t_{9}^{l m} .
$$

Using the explicit forms of the coefficients we obtain

$$
t^{l m}=\frac{\mu}{r^{2}} \delta(r-R(t))\left(E v_{r}^{2} e^{4 \lambda}-E+e^{2 \nu} \frac{L^{2}}{r^{2} E}\right) Y_{l m}^{*},
$$

and by making use of the geodesic equation (3b) we can reduce this expression to

$$
t^{l m}=-e^{2 \nu} \frac{\mu}{r^{2} E} \delta(r-R(t)) Y_{l m}^{*} .
$$

[1] K.S. Thorne and A. Campolattaro, Astrophys. J. 149, 591 (1967) + Erratum, Astrophys. J. 152, 673 (1968).

[2] Y. Kojima, Prog. Theo. Phys. 79, 665 (1988).

[3] K. D. Kokkotas and B. F. Schutz, Mon. Not. R. Astron. Soc. 255, 119 (1992).

[4] M. Leins, H.-P. Nollert, M. H. Soffel, Phys. Rev. D 48, 3467 (1993).

[5] G. Allen, N. Andersson, K.D. Kokkotas, B.F.Schutz Phys. Rev. D 58, 124012 (1998).

[6] J. Ruoff, submitted to Phys. Rev. D, archived under gr-qc/0003088

[7] G. Allen, N. Andersson, K.D. Kokkotas, P. Laguna, J. Pullin and J. Ruoff, Phys. Rev. D 60, 104021 (1999).

[8] N. Andersson and K.D. Kokkotas, Mon. Not. R. Astron. Soc. 299, 1059 (1998). 
[9] A. Borelli, Nuovo Cimento B 112, 225 (1997).

[10] V. Ferrari, L. Gualtieri, and A. Borelli, Phys. Rev. D 59, 124020 (1999).

[11] K. Tominaga and M. Saijo, Phys. Rev. D 60, 024004 (1999).

[12] Z. Andrade and R. Price, Phys. Rev. D 60, 104037 (1999).

[13] R. Arnowitt, S. Deser, and C.W. Misner, in Gravitation: An Introduction to Current Research, edited by L. Witten (John Wiley, New York, 1962), pp. 227-265

[14] T. Regge and J.A. Wheeler, Phys. Rev. 108, 1063 (1957).

[15] F.J. Zerilli, J. Math. Phys. 11, 2203 (1970).

[16] C. Cutler, L.S. Finn, E. Poisson and G.J. Sussman, Phys. Rev. D 47, 1511 (1993).

[17] Y. Kojima, Prog. Theo. Phys. 77, 297 (1987).

[18] F.J. Zerilli, Phys. Rev. D 2, 2141 (1970).

[19] J. Ruoff, PhD Thesis, Universität Tübingen, 2000 to be published.
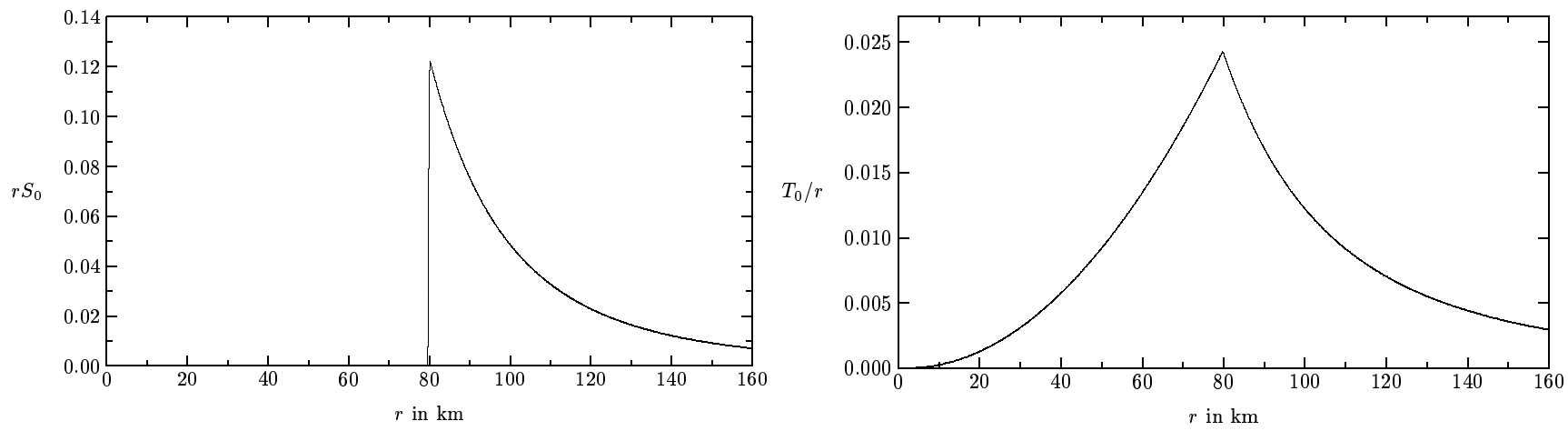

FIG. 1. Profile of the initial values for both kind of initial data. The left panel shows $S_{0}$ when $T_{0}=0$. The right panel shows $T_{0}$ when $S_{0}=0$. Note, that $S$ exhibits a discontinuity at the particle's location whereas $T$ is continuous. 

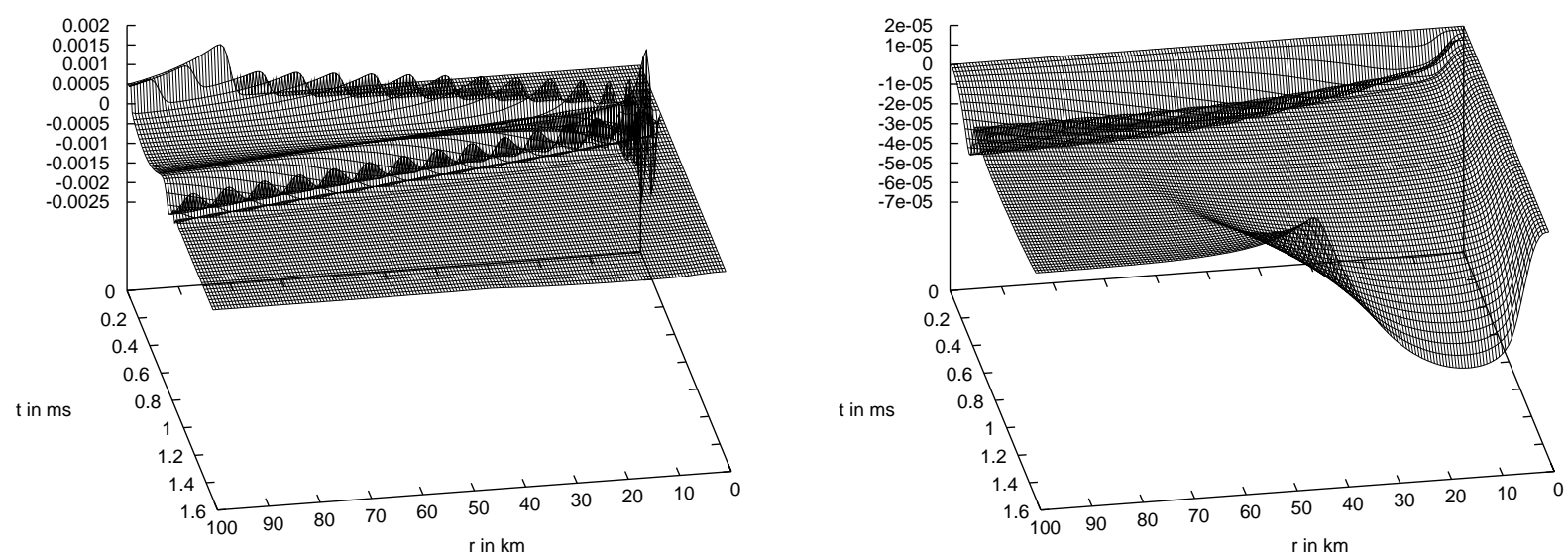

FIG. 2. Evolution of $S$. The left panel shows the evolution for $T_{0}=0$, in the right panel $S_{0}=0$. In the first case shows a burst of gravitational waves that propagate both in and outwards. The ingoing pulse gets reflected at the origin and travels back outwards again. In the other case, a wave originating from the interior of the neutron star travels outwards. Note the differences in the amplitudes of both cases.
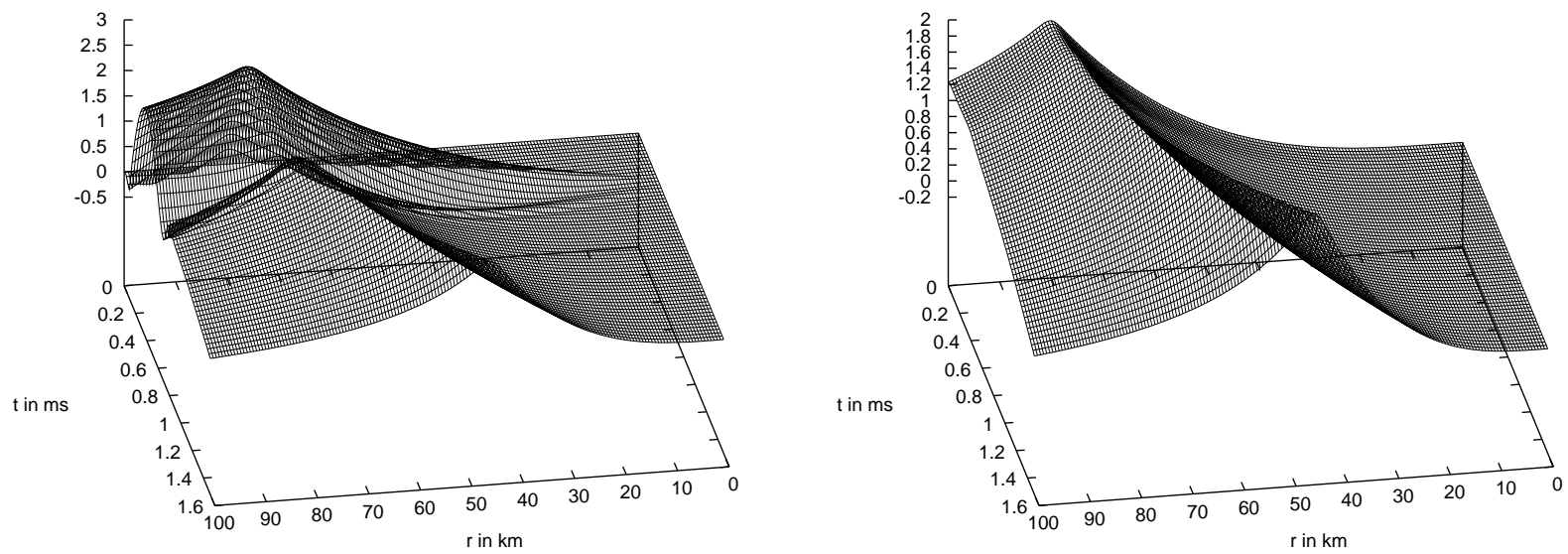

FIG. 3. Evolution of $T$. The left panel shows the evolution for $T_{0}=0$, in the right panel $S_{0}=0$. In the first case, we can see the built up of $T$ which is disturbed by the reflected part of the wave that was sent out by the particle. The second case clearly shows that $T$ basically has its "right" shape right from the beginning.
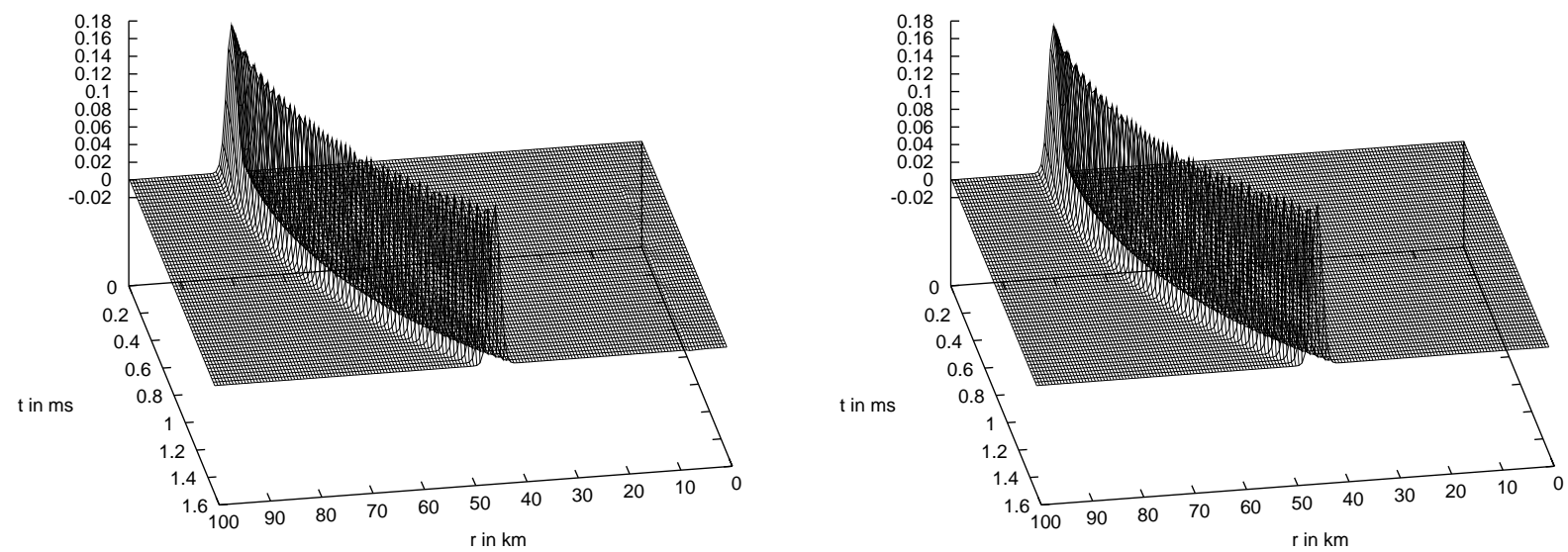

FIG. 4. Evaluation of the Hamiltonian constraint during the evolution. Again, the left panel shows the evolution for $T_{0}=0$, in the right panel $S_{0}=0$. The Gaussian shape of the particle is clearly visible and has been chosen to be very broad for a better visualization. The particle is initially at rest and starts falling towards the neutron star. 

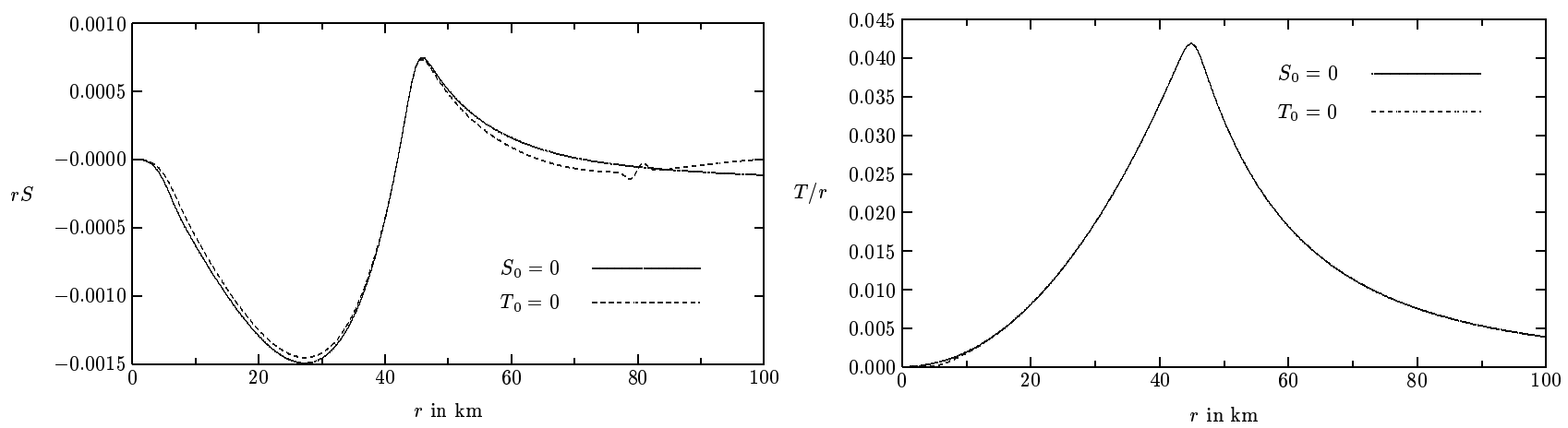

FIG. 5. Plot of the variables $S$ and $T$ at the end of the evolution of the different initial data. The artificial radiation of the initial data has been radiated away and both variables have assumed their "right" values that is independent of the initial data. The difference in $S$ is due to the fact that the initial data with $T_{0}=0$ contain much more radiation which excites the neutron stars to pulsations which in turn disturb the profile of $S$. The variable $T$ is mostly unaffected by this.
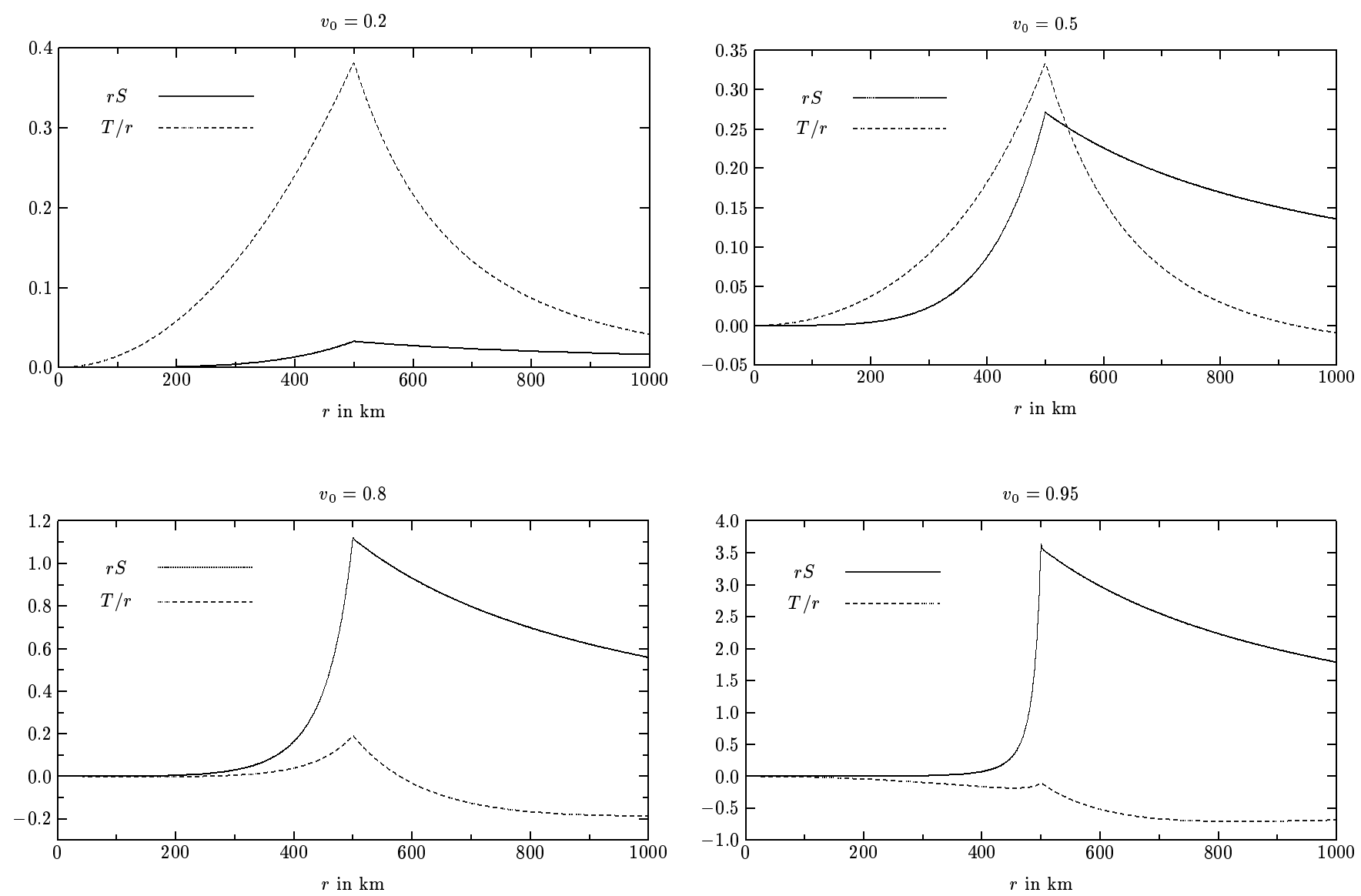

FIG. 6. Initial data for a particle with different initial radial velocities $v$ where $S$ is given by (39) and $T$ is obtained by solving (38). For small velocities, $T / r$ dominates $r S$ whereas for ultra-relativistic velocities, $r S$ dominates $T / r$. 


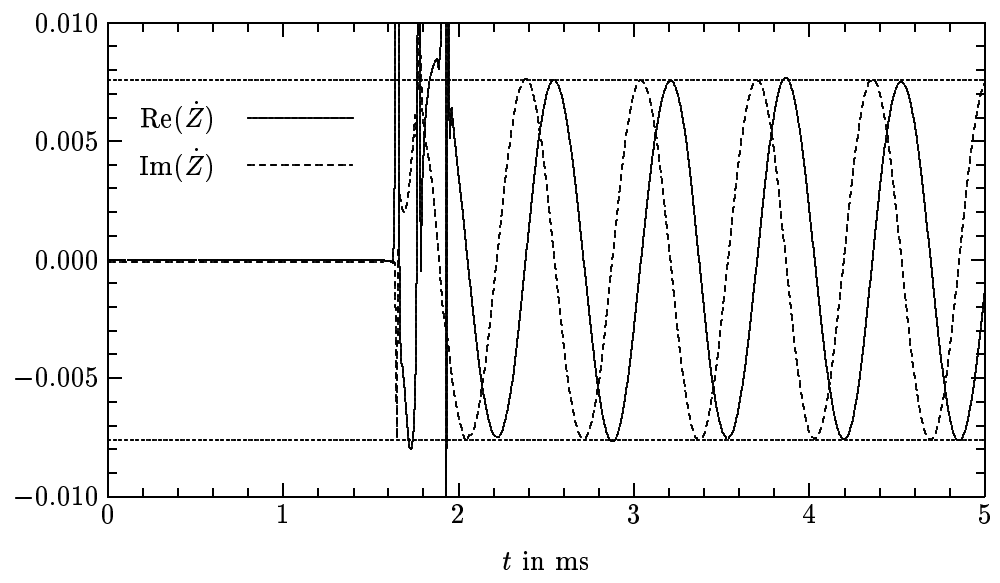

FIG. 7. Evolution of the real and imaginary parts of $\dot{Z}_{22}$ at $r=500 \mathrm{~km}$. After the initial radiation bursts the waveforms show periodical oscillations with twice the orbital frequency. The amplitude is about 0.0076 which corresponds to a radiation power of $(M / \mu)^{2} d E_{22} / d t=5.46 \times 10^{-5}$.
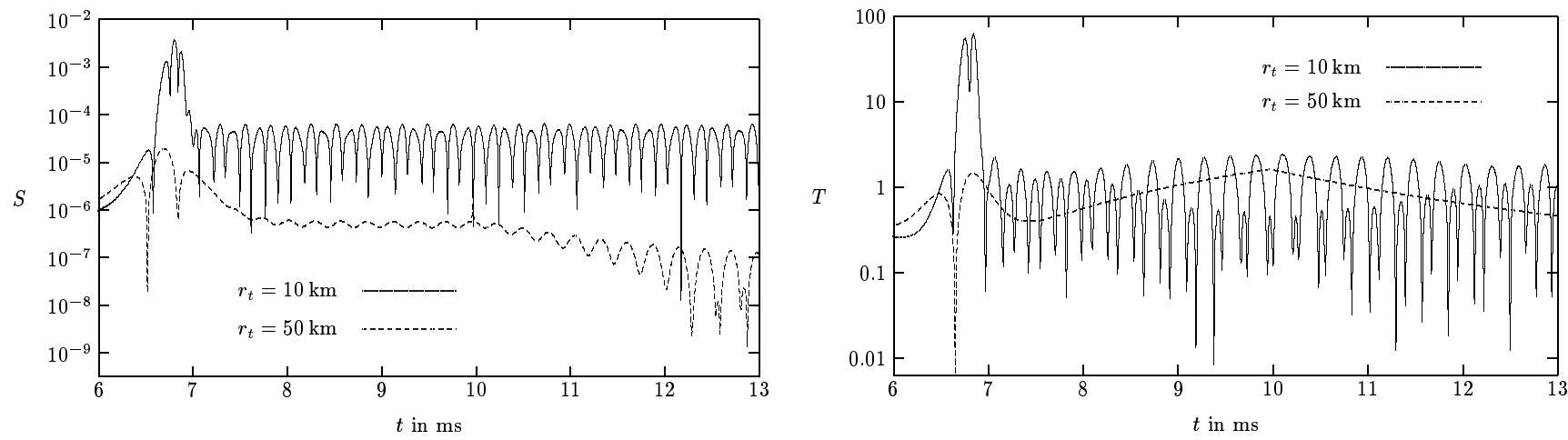

FIG. 8. shows the difference of excitation strengths for the two different turning radii $r_{t}=10 \mathrm{~km}$ (solid line) and $r_{t}=50 \mathrm{~km}$ (dashed line). The waveform of $S$ (left panel) for $r_{t}=10 \mathrm{~km}$ is totally unaffected by the presence of the particle whereas for $r_{t}=50 \mathrm{~km}$, the gravitational field shifts the amplitude of the signal to higher values. For $T$ this effect is much more pronounced and can already be detected for $r_{t}=10 \mathrm{~km}$. For $r_{t}=50 \mathrm{~km}$, the oscillations of the neutron star are totally buried in the gravitational field of the particle. At $t=10 \mathrm{~ms}$ the particle crosses the observer who is located at $r_{o b s}=1000 \mathrm{~km}$. 

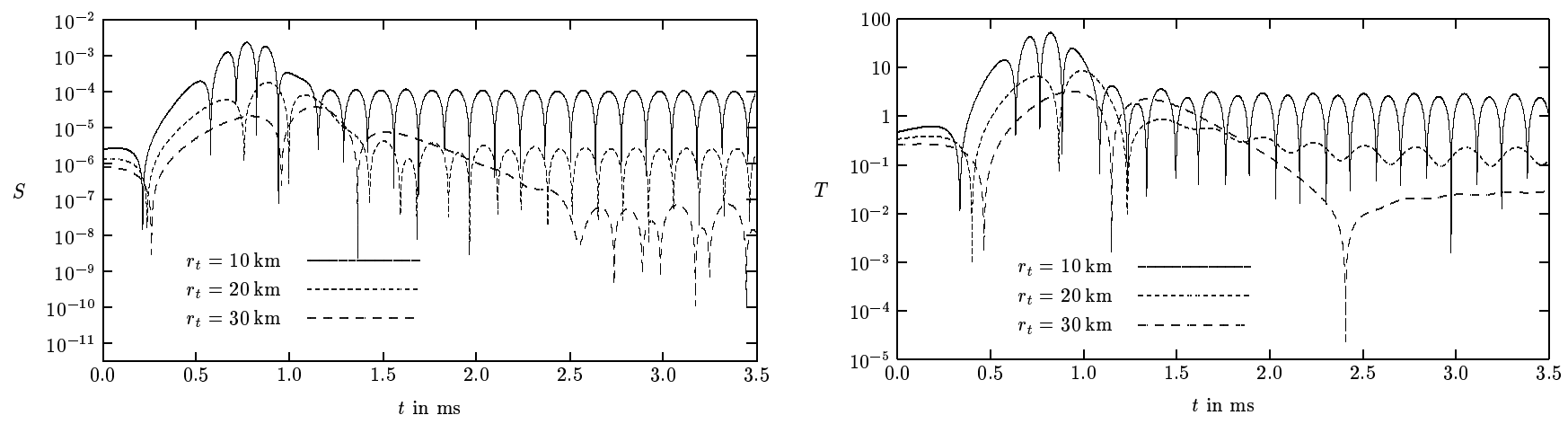

FIG. 9. Waveforms of $S$ and $T$ for the three different turning radii $r_{t}=10 \mathrm{~km}, r_{t}=20 \mathrm{~km}$ and $r_{t}=30 \mathrm{~km}$. The initial velocity of the particle is $v_{0}=-0.1$
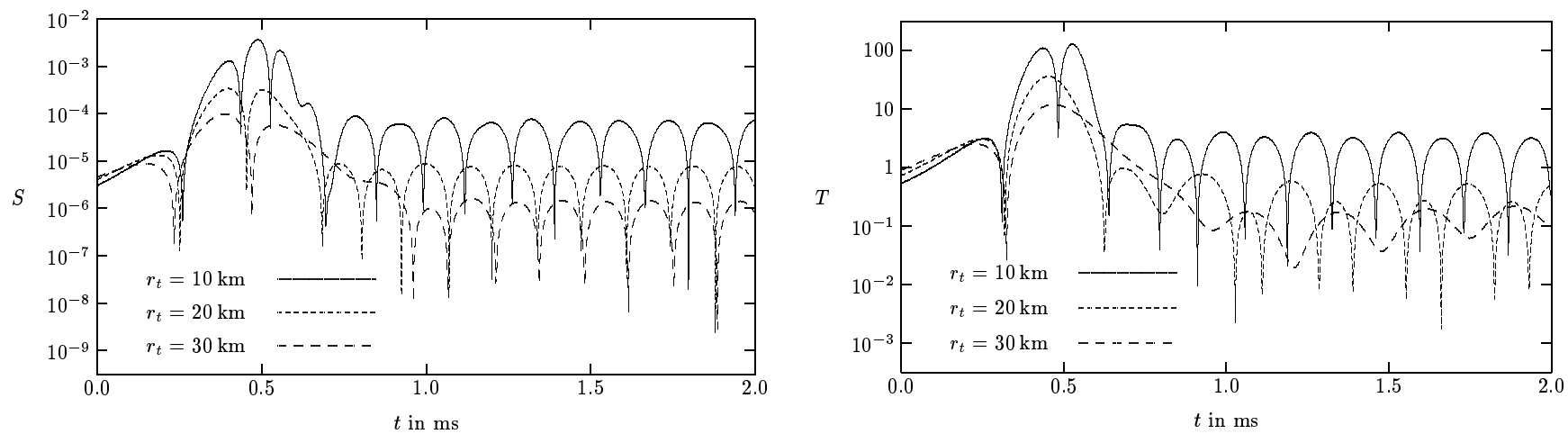

FIG. 10. Waveforms of $S$ and $T$ for the three different turning radii $r_{t}=10 \mathrm{~km}, r_{t}=20 \mathrm{~km}$ and $r_{t}=30 \mathrm{~km}$. The initial velocity of the particle is $v_{0}=-0.5$
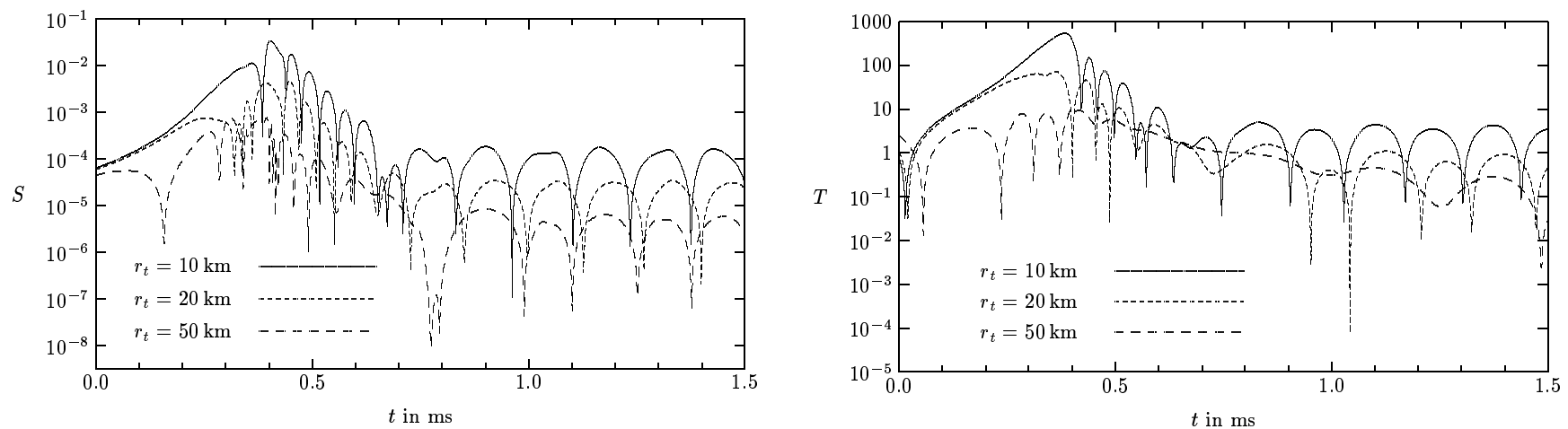

FIG. 11. Waveforms of $S$ and $T$ for the three different turning radii $r_{t}=10 \mathrm{~km}, r_{t}=20 \mathrm{~km}$ and $r_{t}=50 \mathrm{~km}$. The initial velocity of the particle is $v_{0}=-0.97$ 

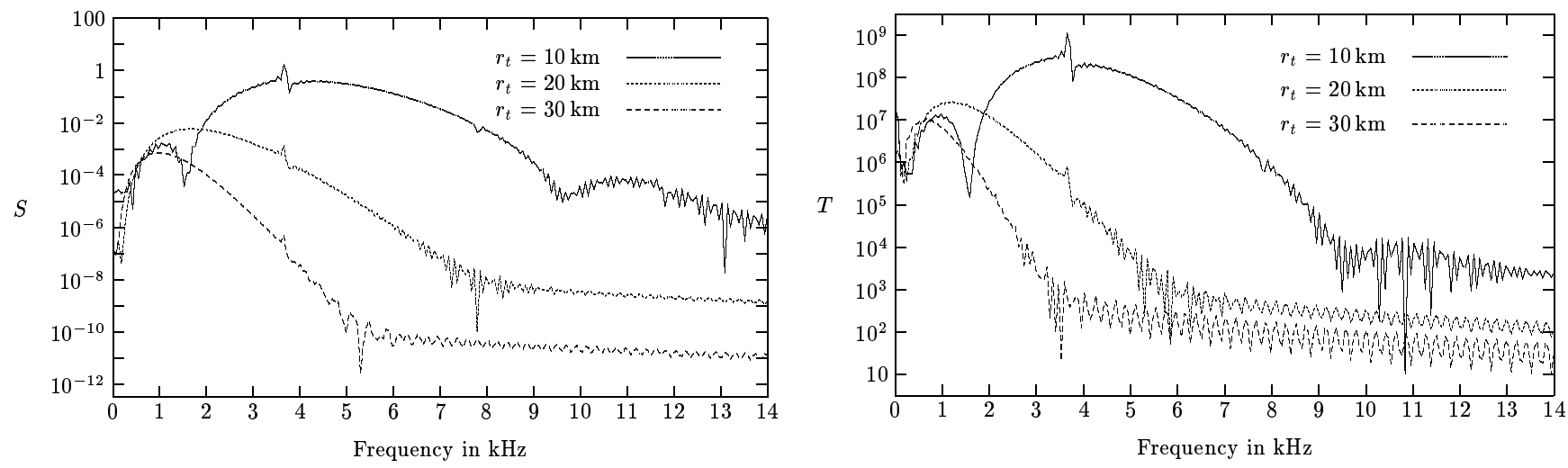

FIG. 12. Fourier transform of the waveforms for $v_{0}=-0.1$
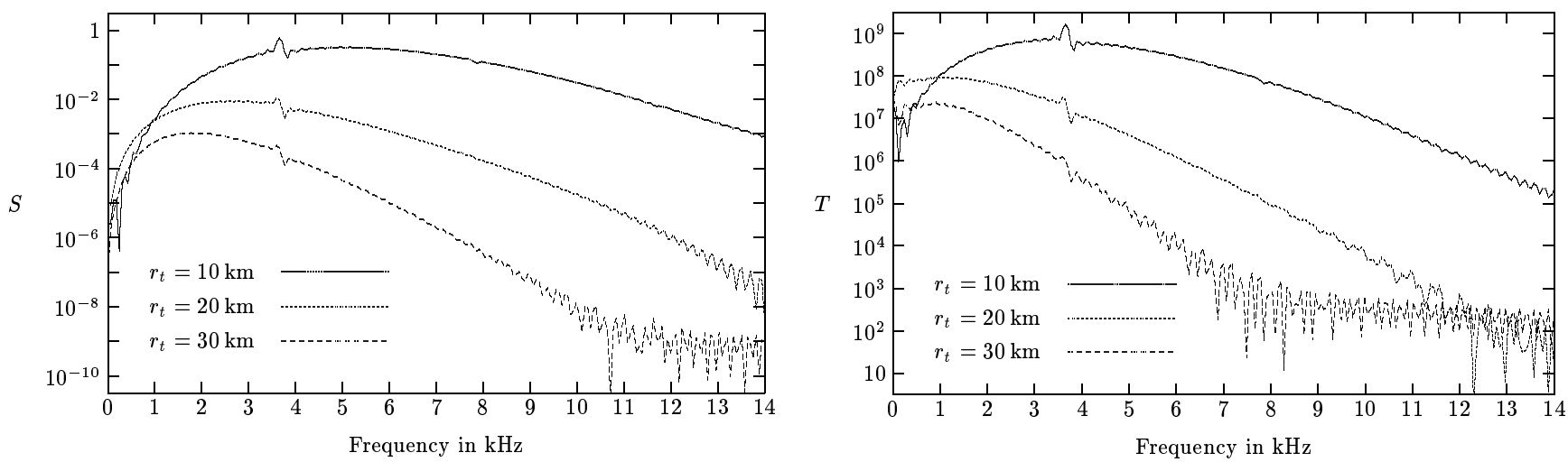

FIG. 13. Fourier transform of the waveforms for $v_{0}=-0.5$
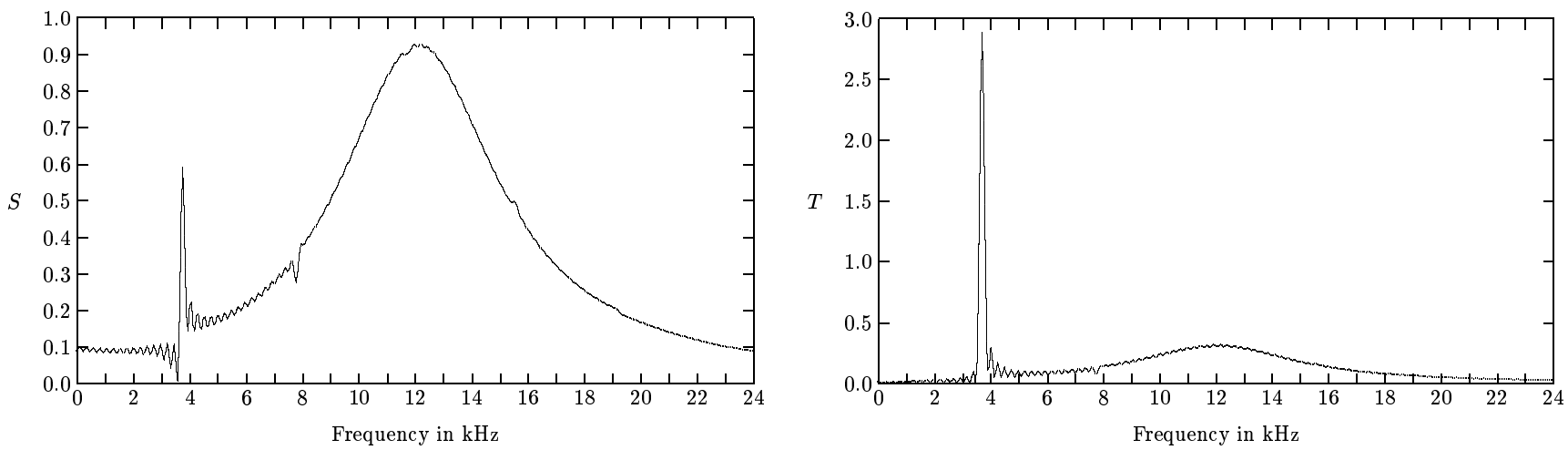

FIG. 14. Fourier transform of the waveform for $r_{t}=10 \mathrm{~km}$ and $v_{0}=-0.97$. The presence of the first $w$-mode is clearly visible. 